\title{
Clinical features of post-infectious bronchiolitis obliterans in children undergoing long-term azithromycin treatment
}

\author{
XUEYAN WANG ${ }^{1}$, CHANGSHAN LIU $^{1}$, MENGJUAN WANG ${ }^{1}$, YI ZHANG ${ }^{1},{\text { HEWEN } \text { LI }^{2} \text { and GELI LIU }}^{3}$ \\ Departments of ${ }^{1}$ Pediatrics and ${ }^{2}$ Radiology, The Second Hospital of Tianjin Medical University, Heping, Tianjin 300211; \\ ${ }^{3}$ Department of Pediatrics, The General Hospital of Tianjin Medical University, Heping, Tianjin 300052, P.R. China
}

Received September 26, 2014; Accepted March 5, 2015

DOI: 10.3892/etm.2015.2418

\begin{abstract}
The aim of the present study was to outline any predisposing factors and clinical and radiological features of post-infectious bronchiolitis obliterans (PIBO) in pediatric patients, and to determine the effect of long-term azithromycin treatment on these factors. In total, 16 cases of children with PIBO were retrospectively reviewed. Adenovirus and Mycoplasma pneumoniae were the most common etiological agents (12/16) in the children with PIBO. The patients presented with persistent dyspnea, a chronic cough, sputum production and wheezing following the initial lung infection. Chest X-rays indicated pulmonary overinflation and patchy ground-glass opacity. In addition, high-resolution computed tomography (HRCT) scans revealed patchy ground-glass opacity, bronchiectasis, bronchial wall thickening and mosaic perfusion in all 16 cases. A unilateral hyperlucent lung was observed in two cases. All the patients underwent treatment with low-dose azithromycin and prednisone. Follow-up examinations of the 16 cases, varying in duration between 7 and 31 months, showed that the disease condition had improved in 10 cases. However, no significant improvements were identified from the HRCT scans or were observed in the patient condition in the additional six cases. The diagnosis of $\mathrm{BO}$ is primarily based on a typical clinical presentation and HRCT observations. Therefore, a typical clinical history and patchy ground-glass opacity features on HRCT scans are screening indices that predict BO development. Steroids are the cornerstone of BO treatment; however, long-term azithromycin treatment can improve the condition of the patients. In summary, PIBO is a disease with a high morbidity rate and should be treated by a multidisciplinary team. Patients should receive follow-up examination for an extended period. Patchy ground-glass opacity features on HRCT scans indicate that
\end{abstract}

Correspondence to: Dr Geli Liu, Department of Pediatrics, The General Hospital of Tianjin Medical University, 154 Anshan Road, Heping, Tianjin 300052, P.R. China

E-mail: geliliucc@126.com

Key words: post-infectious bronchiolitis obliterans, child, azithromycin, steroids clinical suspicion of BO is necessary in children with persistent and severe wheezing.

\section{Introduction}

Bronchiolitis obliterans (BO) is an inflammatory disease of the small airways that is caused by an insult to the lower respiratory tract. The presence of inflammation and fibrosis of the terminal and respiratory bronchioles leads to a narrowing or full obliteration of the airway lumen, and subsequently to the chronic obstruction of airflow. BO is characterized by the presence of intraluminal granulation tissue in the airways or peribronchial fibrosis and lumen narrowing, resulting in scarring and obstruction (1).

BO is diagnosed according to a variety of factors, including the history of insult to the lower respiratory tract (usually caused by infection), persistent symptoms that do not respond to the administration of bronchodilators for two weeks, computed tomographic (CT) findings and the exclusion of other diseases (2). However, BO is often undiagnosed or misdiagnosed as other childhood lung diseases, such as wheezing syndromes.

Epidemiological factors, treatment and disease prognosis should be clearly defined in order to reduce the morbidity and mortality rates of the disease. The aim of the present study was to describe the clinical and radiological findings, and the treatment protocols, during the follow-up of patients with BO in the Pediatric Department of the Second Hospital of Tianjin Medical University (Tianjin, China).

Increasing evidence has demonstrated that macrolide antibiotics exert immune-modifying properties, in addition to their role as antimicrobials (3). Azithromycin has been reported to be effective for long-term use in the treatment of several chronic conditions, including mycobacterial diseases, cystic fibrosis and asthma (4). A previous study demonstrated that following three months of treatment with azithromycin, airway neutrophilia was shown to decrease in patients with BO syndrome (BOS) that had undergone a lung transplantation (5). Therefore, long-term low-dose azithromycin therapy may offer a novel, safe therapy for patients with BOS who have undergone a lung transplantation. To the best of our knowledge, the present study was the first long-term study investigating azithromycin treatment for patients with post-infectious BO (PIBO). 


\section{Subjects and methods}

Subjects. This retrospective study reviewed the clinical records of children diagnosed with PIBO at the Second Hospital of Tianjin Medical University between August 2010 and March 2013. Patient gender, age at diagnosis, imaging features, etiology, clinical course, treatment and prognosis were analyzed. The current study was conducted in accordance with the Declaration of Helsinki and with approval from the Ethics Committee of the Second Hospital of Tianjin Medical University. Written informed consent was obtained from the parents/guardians of the participants.

Clinical criteria. The clinical criteria for a diagnosis of PIBO were as follows (6): i) Persistent dyspnea for $>6$ weeks following an acute infection or acute lung injury; ii) unresolved wheezing or a cough with no reaction to bronchiectasis agents; iii) respiratory symptoms that were severely disproportionate to the chest $X$-ray findings; iv) bronchial wall thickening, bronchiectasis, pulmonary atelectasis or mosaic perfusion, as observed on a pulmonary high-resolution CT (HRCT) scan; v) obstructive ventilatory disorder observed using lung function tests; vi) unilateral hyperlucent lung observed via a chest radiography examination; and vii) exclusion of other obstructive pulmonary pathologies, such as asthma, primary ciliary dyskinesia syndrome, immune deficiency or pancreatic fibrocystic changes.

\section{Results}

Clinical features. A total of 16 children with PIBO were identified. All 16 cases occurred following moderate-severe pneumonia. Table I presents the clinical features of the patients with PIBO. Of the 16 cases, 13 were male and three were female. The median age of the patients was 20.0 months, and ranged between 3.0 months and 6.0 years. The mean hospital stay was 28.25 days (range, 15-57 days). The initial clinical manifestations were repeated coughing, wheezing and tachypnea following a respiratory infection. The 16 patients exhibited varying degrees of tachypnea; moist rales and wheezing were heard in the lung field. A severe cough and wheezing were observed in 12 patients at the time of admission. In addition, 14 patients had a high fever, and six patients had a transcutaneous blood oxygen saturation of $86-91 \%$. The children had no family history of asthma. The disease course varied between 7 and 31 months.

Etiological agents. The initial event that led to BO in the majority of the children was pneumonia. The causal agent in 13 of the cases was identified using an immunofluorescence detection kit (Thermo Fisher Scientific, Waltham, MA, USA) on nasal-pharynx secretions at initial events or by determining specific serum antibodies during the later stages of the disease using a Mycoplasma pneumoniae antibody ELISA kit (Shanghai Tong Wei Industrial Co., Ltd.). The causal agent in three cases remained unknown. Adenovirus and Mycoplasma pneumoniae were the most common etiological agents (12/16), with six cases caused by adenovirus, six cases associated with Mycoplasma pneumoniae, and one case associated with Epstein-Barr virus.
Imaging findings. Chest X-rays, performed using a SOMATOM Definition 64 CT scanner (Siemens Healthcare, Erlangen, Germany), revealed that 12 patients had excessive ventilation and 10 patients exhibited patchy ground-glass opacity. The HRCT scans showed patchy ground-glass opacity and mosaic perfusion in all 16 cases; bronchial wall thickening and bronchiectasis were observed in eight cases; pulmonary atelectasis was noted in two cases; and a unilateral hyperlucent lung was observed in two cases (typical cases are shown in Figs. 1 and 2). Lung perfusion scans were performed in two patients and revealed segmental perfusion defects, which correlated with the abnormalities observed on the chest radiographs.

Treatment following discharge. When discharged, the patients received supportive treatment, which included inhaled bronchodilators, chest physiotherapy and empiric antibiotics when required for acute respiratory infections. Systemic corticosteroid therapy (prednisolone, $1 \mathrm{mg} / \mathrm{kg} / \mathrm{day}$; Tianjin Lisheng Pharmaceutical Co., Ltd., Tianjin, China) and azithromycin (7.5 mg/kg, twice weekly; Hisun-Pfizer Pharmaceuticals Ltd., Shanghai, China) were used administered to all 16 patients with a dose reduction of $1.25 \mathrm{mg} / \mathrm{month}$. The treatment period for azithromycin and prednisone therapy ranged between 6 and 27 months, and the patients underwent follow-up examinations for various time periods, ranging between 7 and 31 months. Clinical symptoms and HRCT observations were significantly improved in 10 cases, whereas the HRCT features and patient condition showed no significant improvement in four cases. Six months after discharge, the clinical symptoms became more serious in two patients, who discontinued the prednisone therapy; HRCT scans revealed a unilateral hyperlucent lung.

\section{Discussion}

BO is a chronic obstruction of the airflow associated with inflammatory lesions of the small airways (1). The condition is divided into two types depending on the basis of the histological features, namely proliferative bronchiolitis and obstructive or constrictive bronchiolitis. The latter form is predominant (7). Constrictive bronchiolitis has a range of morphological abnormalities, from bronchiolar to peribronchiolar inflammation, and the condition can lead to complete obstruction of the bronchioles through submucosal fibrosis (8).

The clinical manifestations of BO are usually continuous or repeated coughing and wheezing, and a poor exercise tolerance. Distinguishing BO from ordinary bronchiolitis or viral pneumonia through observation of the symptoms is challenging; however, delaying treatment may result in aggravated respiratory tract infections or even mortality due to respiratory failure in a period of one to two years (2).

$\mathrm{BO}$ has a number of causes, including infection, connective tissue disorders, bone marrow or lung transplantation and severe mucocutaneous allergic disorders, such as Stevens-Johnson syndrome, or the inhalation of toxic substances (9-11). BO following a severe infectious lung disease is the most common form reported in children (12). The causal agents of BO include adenovirus, influenza virus, measles virus and Mycoplasma pneumoniae (13). Adenovirus is the leading infectious cause of BO worldwide (14). In the present study, $37.5 \%$ of patients $(6 / 16)$ were infected with adenovirus. An 


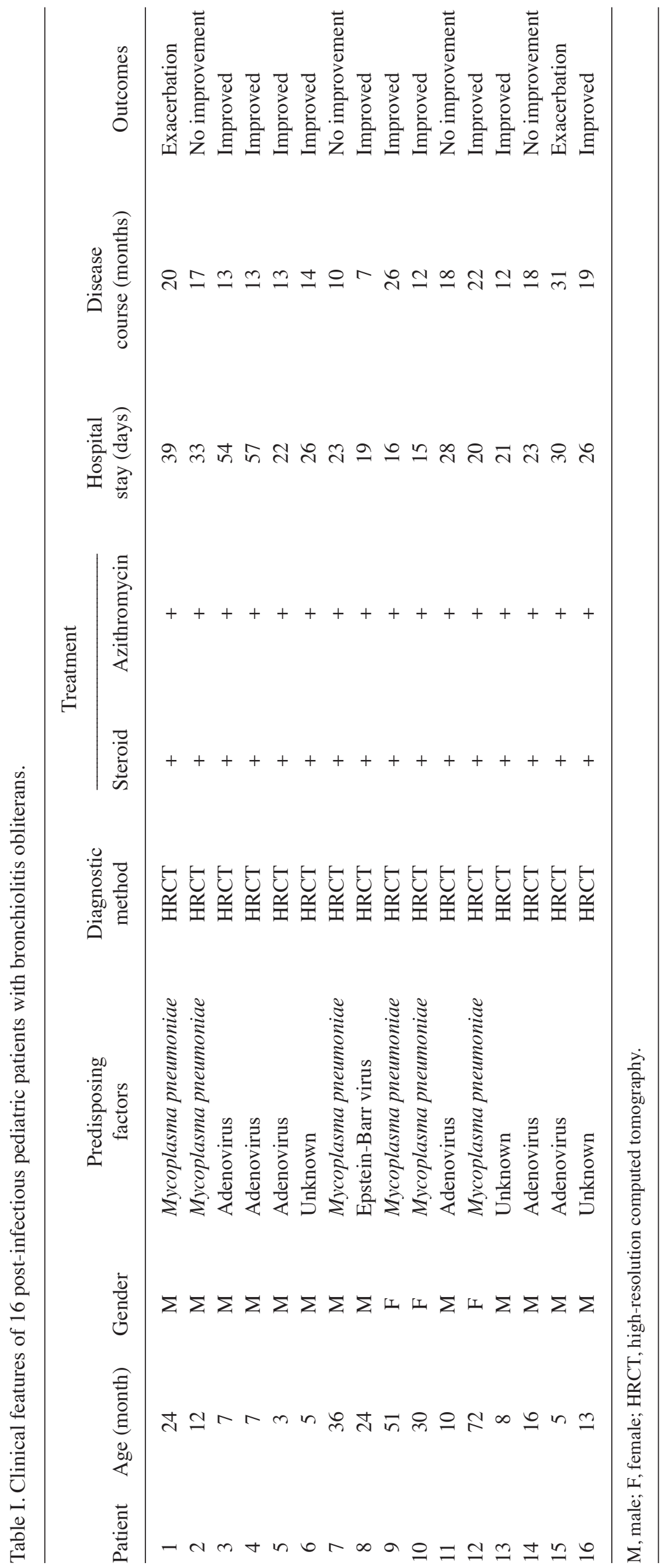


A

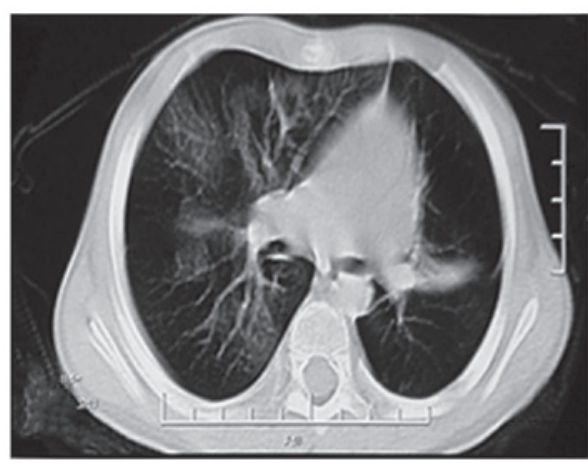

B

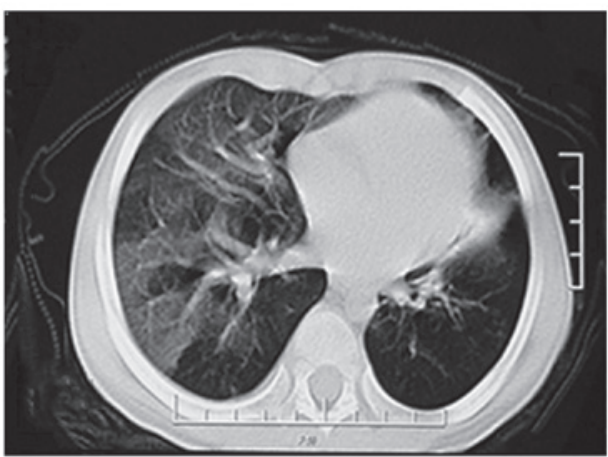

Figure 1. Images of case 1, a two-year-old male patient, were captured at the 6-month follow-up. (A) and (B) display the same CT scan. High-resolution computed tomography scans show areas of consolidation in the left lung field and mosaic ground-glass patterns with air trapping in the right lung. Note the small size of the left lung (unilateral hyperlucent lung, Swyer-James syndrome).

A

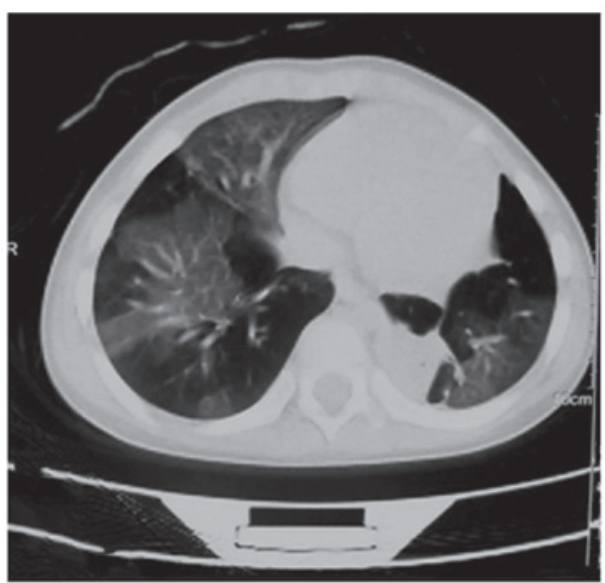

B

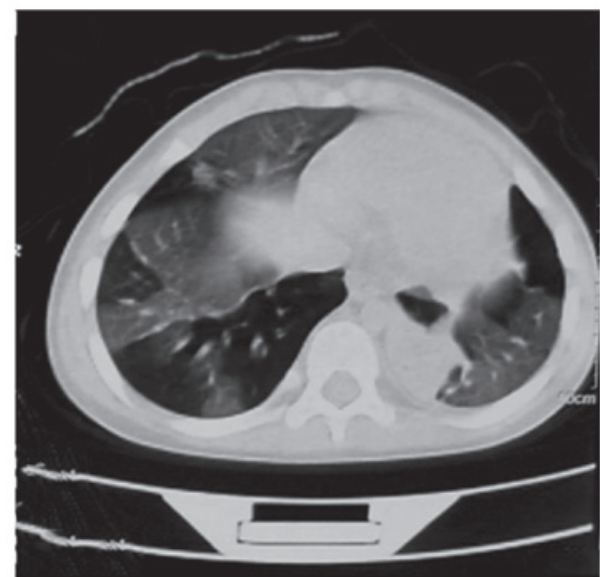

Figure 2. Images of case 2, a one-year-old male patient, were captured at the 15-month follow-up. (A) and (B) display the same CT scan. High-resolution computed tomography images show the small size of the left lung and bilateral mosaic ground-glass patterns with air trapping. The images show the collapse of the left lung with atelectasis and consolidation.

additional major infectious agent is Mycoplasma pneumoniae, which accounted for $37.5 \%$ of patients in the present study. The increased percentage of cases with Mycoplasma pneumoniae as a causal agent may be associated with the increased incidence of Mycoplasma pneumonia in China.

Respiratory signs and symptoms of acute viral bronchiolitis disappear within a few days. Therefore, in children with acute lower respiratory tract infections, BO must be considered if wheezing and respiratory distress do not resolve within the expected time frame, particularly in those with severe adenovirus infections. Further diagnosis is imperative (15).

With regard to diagnosis, PIBO has no specific signs and symptoms. Ideally, the diagnosis requires histopathological confirmation; however, the irregular distribution of the lesions results in sample collection being challenging (16). Therefore, clinical and imaging criteria are combined with laboratory tests for agent identification and to eliminate other forms of chronic lung disease. Imaging techniques, particularly HRCT, play an important role in the diagnosis of PIBO $(17,18)$. A number of characteristics are observed on PIBO-positive HRCT scans, including patchy ground-glass opacity, air retention, bronchial wall thickening, bronchiectasis, mosaic perfusion and a unilateral hyperlucent lung (19). Air retention syndrome has the highest sensitivity and accuracy in the diagnosis of BO.
Leung et al (20) reported that the sensitivity of air retention syndrome in HRCT was $91 \%$, with a specificity of $80 \%$ and an accuracy of $86 \%$. The expiratory phase of respiration is important in diagnosing air retention, particularly in less severe cases where the inspiratory phase may miss or underestimate this important characteristic (21). Acquiring scans of the two respiratory phases may be a challenge in uncooperative pediatric patients; therefore, more attention should be paid to ground-glass opacity features. In the current study, the HRCT results from 10 patients revealed ground-glass opacity.

In the study by Colom and Teper (22), the following scoring method was proposed: Typical medical history (4 points); adenovirus infection ( 3 points) and HRCT showing mosaic perfusion syndrome (4 points). A score of $>7$ points indicated a diagnosis of BO, with a specificity of $100 \%$ and a sensitivity of $67 \%$.

PIBO is a rare disease, which has limited the opportunity for proper randomized clinical trials focused on its treatment; thus, therapeutic decisions are empirically based $(23,24)$. The drug treatments for PIBO include: i) Oral or inhaled corticosteroids aimed at reducing the inflammatory component; ii) hydroxychloroquine and high-dose pulses of methylprednisolone for treating severe or prolonged obstruction; iii) short and long-acting bronchodilators or inhaled anticholinergic 
agents for cases of symptomatic wheezing; and iv) antibiotics, orally or intravenously administered, for the treatment of patients with frequent infections (25).

Oxygen supplementation may also be used in addition to drug treatment, particularly during the first few years of the disease. In the majority of cases, subsequent clinical improvement leads to the complete weaning off of oxygen. The requirement for supplemental oxygen at night is a concern; however, only in severe cases have patients demonstrated significant desaturation during sleep (26).

Although inflammation plays a prominent role in the pathogenesis of $\mathrm{BO}$, the use of corticosteroid drug treatment remains controversial due to the side effects. Certain studies have suggested that steroids may slow down the progression of bronchiole fibrosis (27). In the current study, the patients were administered an initial dose of prednisone of $1 \mathrm{mg} / \mathrm{kg} / \mathrm{day}$. The dose was gradually reduced after 3 months, with the overall course duration varying between 6 and 27 months, or longer. A previous study used steroid therapy, for a period ranging between 1 and 60 months, in children receiving different BO treatments, including bronchodilators, azithromycin and oxygen therapy (28). Among these patients, $>80 \%$ exhibited rapid improvement in disease symptoms when medicated using steroids.

Azithromycin is a macrolide antibiotic that has been demonstrated in prospective, double-blind, placebo-controlled trials to be effective for the treatment of diffuse panbronchiolitis and cystic fibrosis. The efficacy of azithromycin has been hypothesized to be a result of its anti-inflammatory effects (29).

In addition, a previous study in patients with BOS following a lung transplant have demonstrated an improvement in the forced expiratory volume in $1 \mathrm{sec}$ following a prolonged course of oral azithromycin $(250 \mathrm{mg}$ three times per week) (30). Although no previous studies have been performed with azithromycin in children with PIBO, oral azithromycin should be considered as a therapeutic option for these patients due to the efficacy demonstrated in the present study.

In conclusion, the results of the current study indicate that a typical clinical history and patchy ground-glass opacity features on HRCT scans can be used as screening indices to predict BO development. Steroid therapy is the cornerstone of BO treatment; however, azithromycin is also indispensable in the treatment of this disease.

\section{References}

1. Lino CA, Batista AK, Soares MA, et al: Bronchiolitis obliterans: clinical and radiological profile of children followed-up in a reference outpatient clinic. Rev Paul Pediatr 31: 10-16, 2013 (In English and Portuguese).

2. Champs NS, Lasmar LM, Camargos PA, Marguet C, Fischer GB and Mocelin HT: Post-infectious bronchiolitis obliterans in children. J Pediatr (Rio J) 87: 187-198, 2011.

3. Friedlander AL and Albert RK: Chronic macrolide therapy in inflammatory airways diseases. Chest 138: 1202-1212, 2010.

4. Hickey AJ, Lu D, Ashley ED and Stout J: Inhaled azithromycin therapy. J Aerosol Med 19: 54-60, 2006.

5. Verleden GM, Vanaudenaerde BM, Dupont LJ and Van Raemdonck DE: Azithromycin reduces airway neutrophilia and interleukin-8 in patients with bronchiolitis obliterans syndrome. Am J Respir Crit Care Med 174: 566-570, 2006.

6. Teper A, Fischer GB and Jones MH: Respiratory sequelae of viral diseases: from diagnosis to treatment. J Pediatr (Rio J) 78 (Suppl 2): S187-S194, 2002 (In Portuguese).
7. Lynch JP III, Weigt SS, DerHovanessian A, Fishbein MC, Gutierrez A and Belperio JA: Obliterative (constrictive) bronchiolitis. Semin Respir Crit Care Med 33: 509-532, 2012.

8. Mauad T and Dolhnikoff M; São Paulo Bronchiolitis Obliterans Study Group: Histology of childhood bronchiolitis obliterans. Pediatr Pulmonol 33: 466-474, 2002.

9. de Blic J, Deschildre A and Chinet T: Post-infectious bronchiolitis obliterans. Rev Mal Respir 30: 152-160, 2013 (In French).

10. Weigt SS, DerHovanessian A, Wallace WD, Lynch JP III and Belperio JA: Bronchiolitis obliterans syndrome: the Achilles' heel of lung transplantation. Semin Respir Crit Care Med 34: 336-351, 2013.

11. Dogra S, Suri D, Saini AG, Rawat A and Sodhi KS: Fatal bronchiolitis obliterans complicating Stevens-Johnson syndrome following treatment with nimesulide: a case report. Ann Trop Paediatr 31: 259-261, 2011.

12. Mattiello R, Mallol J, Fischer GB, Mocelin HT, Rueda B and Sarria EE: Pulmonary function in children and adolescents with postinfectious bronchiolitis obliterans. J Bras Pneumol 36: 453-459, 2010 (In English and Portuguese).

13. Hayes D Jr, Mansour HM, Kirkby S and Phillips AB: Rapid acute onset of bronchiolitis obliterans syndrome in a lung transplant recipient after respiratory syncytial virus infection. Transpl Infect Dis 14: 548-550, 2012

14. Vu DL, Bridevaux PO, Aubert JD, Soccal PM and Kaiser L: Respiratory viruses in lung transplant recipients: a critical review and pooled analysis of clinical studies. Am J Transplant 11: 1071-1078, 2011.

15. Murtagh P, Giubergia V, Viale D, Bauer G and Pena HG: Lower respiratory infections by adenovirus in children. Clinical features and risk factors for bronchiolitis obliterans and mortality. Pediatr Pulmonol 44: 450-456, 2009.

16. Jones KD and Urisman A: Histopathologic approach to the surgical lung biopsy in interstitial lung disease. Clin Chest Med 33: 27-40, 2012.

17. Lee JW, Lee KS, Lee HY, et al: Cryptogenic organizing pneumonia: serial high-resolution CT findings in 22 patients. AJR Am J Roentgenol 195: 916-922, 2010.

18. Devakonda A, Raoof S, Sung A, Travis WD and Naidich D: Bronchiolar disorders: a clinical-radiological diagnostic algorithm. Chest 137: 938-951, 2010.

19. Hochhegger B, Irion KL, Marchiori E, Bello R, Moreira J and Camargo JJ: Computed tomography findings of postoperative complications in lung transplantation. J Bras Pneumol 35: 266-274, 2009 (In English and Portuguese).

20. Leung AN, Fisher K, Valentine V, et al: Bronchiolitis obliterans after lung transplantation: detection using expiratory HRCT. Chest 113: 365-370, 1998.

21. Chen DH, Lin YN, Lan SL, et al: Clinical characteristics of bronchiolitis obliterans in pediatric patients. Zhonghua Er Ke Za Zhi 50: 98-102, 2012 (In Chinese).

22. Colom AJ and Teper AM: Clinical prediction rule to diagnose post-infectious bronchiolitis obliterans in children. Pediatr Pulmonol 44: 1065-1069, 2009.

23. Lenney W, Boner AL, Bont L, et al: Medicines used in respiratory diseases only seen in children. Eur Respir J 34: 531-551, 2009.

24. Vega-Briceño LE and Zenteno AD: Clinical guide for diagnosis and care of children and adolescents with post-infectious bronchiolitis obliterans. Rev Chil Enf Resp 25: 141-163, 2009.

25. Yüksel H, Yilmaz O, Urk V, et al: Clinical significance of lung perfusion defects in children with post-infectious bronchiolitis obliterans. Tuberk Toraks 57: 376-382, 2009.

26. Adde FV, Alvarez AE, Barbisan BN and Guimarães BR: Recommendations for long-term home oxygen therapy in children and adolescents. J Pediatr (Rio J) 89: 6-17, 2013.

27. Yalçin E, Doğru D, Haliloğlu M, Ozçelik U, Kiper N and Göçmen A: Postinfectious bronchiolitis in children: Clinical and radiological profile and prognostic factors. Respiration 70: 371-375, 2003.

28. Kim TO, Oh IJ, Kang HW, et al: Temozolomide-associated bronchiolitis obliterans organizing pneumonia successfully treated with high-dose corticosteroid. J Korean Med Sci 27: 450-453, 2012.

29. Vos R, Vanaudenaerde BM, Verleden SE, et al: Anti-inflammatory and immunomodulatory properties of azithromycin involved in treatment and prevention of chronic lung allograft rejection. Transplantation 94: 101-109, 2012.

30. Aguerre V, Castaños C, Pena HG, Grenoville $M$ and Murtagh P: Postinfectious bronchiolitis obliterans in children: clinical and pulmonary function findings. Pediatr Pulmonol 45: 1180-1185, 2010. 